\title{
Economic burden in Medicaid beneficiaries with recently relapsed schizophrenia or with uncontrolled symptoms of schizophrenia not adherent to antipsychotics
}

Dominic Pilon, MA; Charmi Patel, MPH; Marie-Hélène Lafeuille, MA; Maryia Zhdanava, MA; Dee Lin, PharmD, MS; Aurélie Côté-Sergent, MA; Carmine Rossi, PhD; Patrick Lefebvre, MA; and Kruti Joshi, MPH

\section{What is already known about this subject}

- Schizophrenia is associated with a substantial economic burden, and existing literature suggests that relapse may further increase this burden.

- The association of non-adherence to antipsychotics with costs is less clearly established, particularly among patients with uncontrolled symptoms of schizophrenia.

\section{What this study adds}

- In this study, Medicaid beneficiaries with schizophrenia who experienced a recent relapse and those nonadherent to antipsychotic therapy with uncontrolled symptoms of schizophrenia incurred significantly higher healthcare resource utilization and costs compared to Medicaid beneficiaries without schizophrenia.

- Additional healthcare costs of these two subgroups of patients with schizophrenia appeared higher than those incurred by the overall population with schizophrenia.

\section{Author affiliations}

Dominic Pilon, MA; Marie-Hélène Lafeuille, MA; Maryia Zhdanava, MA; Aurélie CôtéSergent, MA; Carmine Rossi, PhD; Patrick Lefebvre, MA; Analysis Group, Inc., Montréal, Québec, Canada. Charmi Patel, MPH; Dee Lin, PharmD, MS, Kruti Joshi, MPH; Janssen Scientific Affairs, LLC, Titusville, NJ.

\section{AUTHOR CORRESPONDENCE:}

Dominic Pilon, 514.394.4450;

dominic.pilon@analysisgroup.com

J Manag Care Spec Pharm. 2021;27(7):904-14

Copyright $(2021$, Academy of Managed Care Pharmacy. All rights reserved.

APs (proportion of days covered $<80 \%$ ) in the 12-month pre-index period. Previously matched controls were then subset to patients in each subgroup and their matched pairs without schizophrenia, thus maintaining the 1:1 matching ratio. Healthcare resource utilization (HRU) and costs (\$2018 USD) in the 12 -month post-index (observation) period were compared between matched pairs using adjusted regression models

RESULTS: Among 158,763 patients with schizophrenia, 18,771 (11.8\%) had a recent relapse (mean age 50.5 years; $48.6 \%$ female, $51.4 \%$ male) and 13,697 (8.6\%) were not adherent to APs and had uncontrolled symptoms of schizophrenia (mean age 47.1 years; 
48.0\% female, $52.0 \%$ male). During the observation period, patients with recently relapsed schizophrenia and those non-adherent to APs with uncontrolled symptoms of schizophrenia had significantly higher $\mathrm{HRU}$ relative to their controls without schizophrenia. Patients with recently relapsed schizophrenia had mean total healthcare costs $\$ 21,862$ higher relative to their controls ( $\$ 37,424$ vs $\$ 15,563)$, driven by $\$ 8,486$ higher mean long-term care costs (all $P<0.001$ ). Patients non-adherent to APs with uncontrolled symptoms of schizophrenia had adjusted mean total healthcare costs $\$ 20,787$ higher relative to their controls $(\$ 38,337$ vs $\$ 15,241)$, driven by $\$ 8,019$ higher adjusted mean inpatient costs (all $P<0.001$ ). Additional total healthcare costs incurred by patients with recently relapsed schizophrenia and those of patients non-adherent to APs with uncontrolled symptoms of schizophrenia exceeded by $55.2 \%$ and $47.6 \%$, respectively, incremental total healthcare costs incurred by all patients with schizophrenia $(\$ 14,087)$

CONCLUSIONS: Patients with recently relapsed schizophrenia and those non-adherent to AP therapy with uncontrolled symptoms of schizophrenia incurred higher $\mathrm{HRU}$ and costs relative to patients without schizophrenia. Additional healthcare costs of these subgroups of patients with schizophrenia appeared higher than in the overall population with schizophrenia.
2013 analysis of multistate Medicaid claims data, patients with schizophrenia incurred an excess annual mean cost of $\$ 12,447$ relative to controls without schizophrenia. ${ }^{6}$ Similarly, in a more recent study of a six-state Medicaid claims database, patients with schizophrenia incurred \$14,087 higher mean total healthcare costs annually compared to patients without it..$^{10}$ Based on earlier literature, a relapse episode could further increase the burden of schizophrenia and was associated with a mean cost of $\$ 35,725$ per patient in Medicaid beneficiaries. ${ }^{11}$ The cost effect of non-adherence, however, is less clearly established, as a decrease in pharmacy costs among non-adherent patients may potentially offset an increase in hospitalization costs. ${ }^{12}$

The economic burden incurred by specific sub-populations of patients with schizophrenia, such as those with a recent relapse or those who are not adherent to AP therapy with uncontrolled symptoms, has not yet been characterized. Given that it may differ from the economic burden incurred by all schizophrenia patients on average, the objective of the present study was to quantify it using the Medicaid data from six states.

\section{Methods}

Schizophrenia is a chronic and disabling mental illness estimated to affect from $0.25 \%$ to $0.64 \%$ of the US population. ${ }^{1}$ The prevalence of schizophrenia is reported to be higher among Medicaid beneficiaries (1.66\%). ${ }^{2}$

Symptoms of schizophrenia include hallucinations, delusions, and thought disorder, ${ }^{1}$ which can be managed using antipsychotics (APs) if patients remain adherent. ${ }^{3}$ However, many patients have difficulty with long-term adherence to APs and experience detrimental health outcomes as a result, including poor symptom control and relapse. ${ }^{3}$ Schizophrenia relapse is often characterized by a rapid exacerbation of psychoses, increased risk of self-harm and harm to others, and reduced cognitive and social functioning. ${ }^{4,5}$ While schizophrenia is associated with a substantial economic burden, ${ }^{6-8}$ patients who experience a recent disease relapse or those non-adherent to APs with uncontrolled symptoms of schizophrenia may potentially incur even higher healthcare resource use (HRU) and costs. Non-adherence may be associated with uncontrolled symptoms, while incomplete control of symptoms may also prompt patients to discontinue taking medication. ${ }^{9}$ Thus, patients with non-adherence to APs and uncontrolled symptoms of schizophrenia represent a subgroup of particular interest with a potentially large unmet need.

Several prior studies have estimated the economic burden of schizophrenia in various US populations..$^{6-8}$ In a

\section{DATA SOURCE}

This study used Medicaid data from six states, including Iowa (1998Q1-2017Q1), Kansas (2001Q1-2018Q1), Mississippi (2006Q1-2018Q1), Missouri (1997Q1-2018Q1), New Jersey (1997Q1-2014Q1), and Wisconsin (2004Q1-2013Q4). Patient information on enrollment, demographics, medical visits, hospitalizations, long-term care services, prescription drugs, and other services reimbursed by Medicaid were available in the data. Since patient data were de-identified and complied with the Health Insurance Portability and Accountability Act (HIPAA), no institutional review was required.

A retrospective matched cohort design was used (Supplementary Figure 1, available in online article). Initially, cohorts of patients with and without schizophrenia were selected..$^{10}$ Patients with schizophrenia were required to have $\geq 2$ claims with a diagnosis for schizophrenia (International Classification of Diseases, 9th Revision, Clinical Modification [ICD-9-CM]: 295.xx; International Classification of Diseases, 10th Revision, Clinical Modification [ICD-10-CM]: F20.xx, F21, F25.x) on different days. The most recent claim with a diagnosis of schizophrenia, after and before which $\geq 12$ months of continuous Medicaid enrollment were

\section{STUDY DESIGN}




\section{FIGURE 1 Sample Selection}

\section{Patients with schizophrenia}

Patients with $\geq 2$ schizophrenia diagnoses during continuous Medicaid enrollment period

(ICD-9-CM: 295.xx; ICD-10-CM: F20.xx, F21, and F25.x) $\mathrm{N}=312,495$

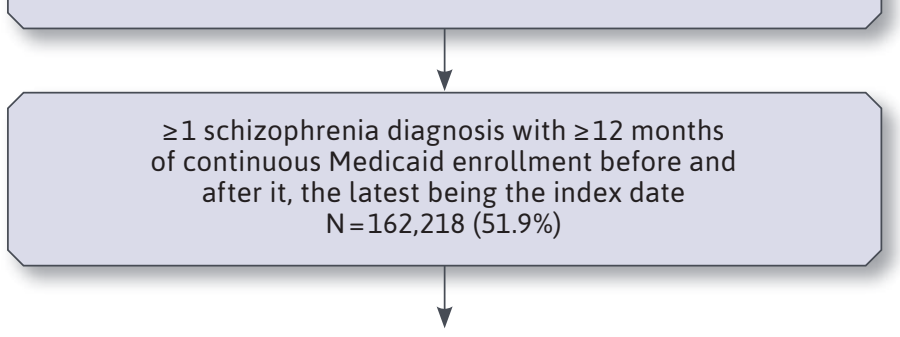

Overall patients with schizophrenia

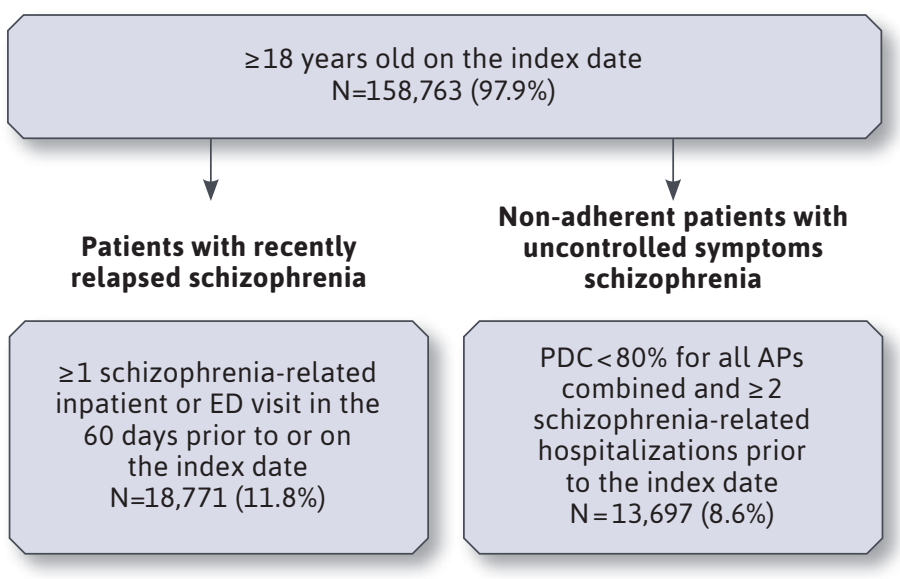

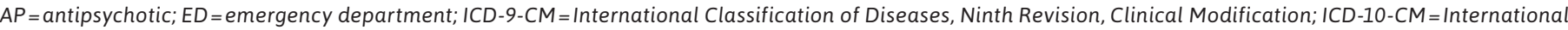
Classification of Diseases, Tenth Revision, Clinical Modification; PDC = proportion of days covered.

\section{Patients without schizophrenia}

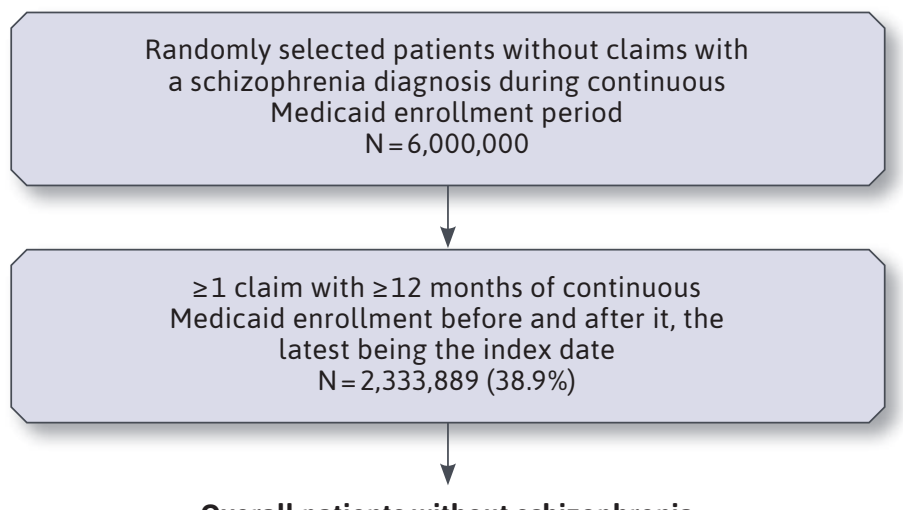

Overall patients without schizophrenia

$\geq 18$ years old on the index date

$\mathrm{N}=1,166,758(50.0 \%)$ available, was defined as the index date. Among patients without schizophrenia, the most recent service claim with $\geq 12$ months of continuous Medicaid enrollment before and after it defined the index date. Both patients with and without schizophrenia were required to be $\geq 18$ years old as of the index date. The two cohorts were matched 1:1 using age groups as an exact matching factor and propensity scores generated based on sex, race, state of residence, eligibility for capitated services and/or dual Medicaid/Medicare coverage, and year of index date. Characteristics included in the matching algorithm were measured during the 12 months preceding the index date (ie, the baseline period, which excludes the index date).

After matching, two subgroups were identified among all adults with schizophrenia. The first included patients with a recent relapse, proxied as $\geq 1$ schizophrenia-related inpatient or emergency department (ED) claim in the 60 days prior to or on the index date. The second included patients not adherent to APs (proportion of days covered [PDC $]<80 \%$; Supplementary Table 1, available in online article) with uncontrolled symptoms of schizophrenia, proxied as $\geq 2$ schizophrenia-related hospitalizations during the baseline period. Previously matched controls were retained for patients in each subgroup, thus maintaining the 1:1 matching ratio. However, given that the propensity score model was only performed to balance characteristics of the initial cohort of patients with schizophrenia, potential imbalances may be present for each subgroup and their matched controls. Outcomes were compared between each subgroup and their controls, respectively. 


\section{TABLE 1}

Baseline Characteristics Among Patients with Recently Relapsed Schizophrenia, Non-Adherent Patients with Uncontrolled Symptoms of Schizophrenia, and Their Matched Controls Without Schizophrenia ${ }^{a}$

\begin{tabular}{|c|c|c|c|c|c|c|}
\hline & $\begin{array}{l}\text { Patients with } \\
\text { recently relapsed } \\
\text { schizophrenia } \\
\mathrm{N}=\mathbf{1 8 , 7 7 1}\end{array}$ & $\begin{array}{c}\text { Patients without } \\
\text { schizophrenia } \\
N=18,771\end{array}$ & $\begin{array}{l}\text { Standardized } \\
\text { difference, \% }\end{array}$ & $\begin{array}{l}\text { Non-adherent } \\
\text { patients with } \\
\text { uncontrolled } \\
\text { symptoms of } \\
\text { schizophrenia } \\
\mathrm{N}=13,697\end{array}$ & $\begin{array}{c}\text { Patients without } \\
\text { schizophrenia } \\
N=13,697\end{array}$ & $\begin{array}{l}\text { Standardized } \\
\text { difference, \% }\end{array}$ \\
\hline $\begin{array}{l}\text { Age at index date, mean } \pm \text { SD } \\
\text { [median; Q1-Q3], years }\end{array}$ & $\begin{array}{c}50.5 \pm 16.3 \\
{[50.7 ; 38.2-61.5]}\end{array}$ & $\begin{array}{c}51.7 \pm 18.5 \\
{[50.7 ; 38.1-61.9]}\end{array}$ & 6.6 & $\begin{array}{c}47.1 \pm 15.4 \\
{[47.2 ; 35.3-57.5]}\end{array}$ & $\begin{array}{c}48.0 \pm 17.4 \\
{[47.4 ; 35.3-57.7]}\end{array}$ & 5.3 \\
\hline \multicolumn{7}{|l|}{ Age categories (years), n (\%) } \\
\hline $18-24$ & $1,102 \quad(5.9)$ & $1,102 \quad(5.9)$ & 0.0 & $1,084 \quad(7.9)$ & $1,084 \quad(7.9)$ & 0.0 \\
\hline $25-34$ & $2,622(14.0)$ & $2,622(14.0)$ & 0.0 & $2,272(16.6)$ & $2,272(16.6)$ & 0.0 \\
\hline $35-44$ & $3,231 \quad(17.2)$ & $3,231 \quad(17.2)$ & 0.0 & 2,779 (20.3) & $2,779(20.3)$ & 0.0 \\
\hline $45-54$ & $4,503(24.0)$ & $4,503(24.0)$ & 0.0 & $3,436(25.1)$ & $3,436(25.1)$ & 0.0 \\
\hline 55-64 & 3,709 (19.8) & 3,709 (19.8) & 0.0 & 2,439 (17.8) & $2,439 \quad(17.8)$ & 0.0 \\
\hline$>65$ & $3,604 \quad(19.2)$ & $3,604 \quad(19.2)$ & 0.0 & $1,687(12.3)$ & $1,687(12.3)$ & 0.0 \\
\hline Female, n (\%) & 9,118 (48.6) & $8,394(44.7)$ & 7.7 & $6,579(48.0)$ & $6,251(45.6)$ & 4.8 \\
\hline \multicolumn{7}{|l|}{ Race, n (\%) } \\
\hline White & $10,789 \quad(57.5)$ & $10,555(56.2)$ & 2.5 & 7,378 (53.9) & 8,069 (58.9) & 10.2 \\
\hline Black & $5,593(29.8)$ & $5,712(30.4)$ & 1.4 & $4,732(34.5)$ & $4,310(31.5)$ & 6.6 \\
\hline Hispanic & $138 \quad(0.7)$ & $131 \quad(0.7)$ & 0.4 & $62 \quad(0.5)$ & $64 \quad(0.5)$ & 0.2 \\
\hline Other & $1,044 \quad(5.6)$ & $947 \quad(5.0)$ & 2.3 & $972 \quad(7.1)$ & $737 \quad(5.4)$ & 7.1 \\
\hline Unknown & $1,207 \quad(6.4)$ & $1,426 \quad(7.6)$ & 4.6 & $553 \quad(4.0)$ & $517 \quad(3.8)$ & 1.4 \\
\hline \multicolumn{7}{|l|}{ State, n (\%) } \\
\hline Missouri & $4,821 \quad(25.7)$ & $4,852(25.8)$ & 0.4 & $5,562(40.6)$ & $5,161 \quad(37.7)$ & 6.0 \\
\hline New Jersey & $5,661(30.2)$ & $5,009 \quad(26.7)$ & 7.7 & $4,881 \quad(35.6)$ & 4,118 (30.1) & 11.9 \\
\hline Wisconsin & 2,463 (13.1) & 2,890 (15.4) & 6.5 & $754 \quad(5.5)$ & $1,290 \quad(9.4)$ & 14.9 \\
\hline Mississippi & 2,147 (11.4) & 2,414 (12.9) & 4.4 & $1,215 \quad(8.9)$ & 1,636 (11.9) & 10.1 \\
\hline lowa & $2,066(11.0)$ & $2,121(11.3)$ & 0.9 & $763 \quad(5.6)$ & $875 \quad(6.4)$ & 3.4 \\
\hline Kansas & $1,613 \quad(8.6)$ & $1,485 \quad(7.9)$ & 2.5 & $522 \quad(3.8)$ & $617 \quad(4.5)$ & 3.5 \\
\hline \multicolumn{7}{|l|}{ Insurance eligibility, n (\%) } \\
\hline Capitated & $3,482(18.5)$ & $4,166(22.2)$ & 9.1 & $1,219 \quad(8.9)$ & $2,476(18.1)$ & 27.1 \\
\hline $\begin{array}{l}\text { Dual Medicaid/Medicare } \\
\text { coverage }\end{array}$ & 11,587 (61.7) & $10,951 \quad(58.3)$ & 6.9 & $6,645(48.5)$ & $6,889 \quad(50.3)$ & 3.6 \\
\hline
\end{tabular}

continued on next page

Outcome Measures. Outcomes were measured during the observation period, which comprised the 12 months on or after the index date. All-cause HRU and healthcare costs were reported over the 12-month observation period and included medical (inpatient visits and days; ED visits; outpatient visits; long-term care [LTC] visits and days; mental health institute $[\mathrm{MHI}]$ admissions, days, and one-day visits; home care days with services; and other service days) and pharmacy components. Additionally, mental health-related HRU and healthcare costs (ie, claims with an ICD-9-CM diagnosis between 290 and 319 [inclusive] or an ICD-10-CM diagnosis between F00 and F99 [inclusive]) and schizophrenia-related HRU and healthcare costs (ie, claims with an ICD-9-CM diagnosis of 295.xx or an ICD-10-CM diagnosis of F20.xx, F21 or F25.x) were also assessed during the observation period. 


\section{TABLE 1 Baseline Characteristics Among Patients with Recently Relapsed Schizophrenia, Non-Adherent Patients with Uncontrolled Symptoms of Schizophrenia, and Their Matched Controls Without Schizophrenia ${ }^{\text {(continued) }}$}

\begin{tabular}{|c|c|c|c|c|c|c|}
\hline & $\begin{array}{l}\text { Patients with } \\
\text { recently relapsed } \\
\text { schizophrenia } \\
\mathrm{N}=18,771\end{array}$ & $\begin{array}{l}\text { Patients without } \\
\text { schizophrenia } \\
\mathrm{N}=18,771\end{array}$ & $\begin{array}{l}\text { Standardized } \\
\text { difference, \% }\end{array}$ & $\begin{array}{l}\text { Non-adherent } \\
\text { patients with } \\
\text { uncontrolled } \\
\text { symptoms of } \\
\text { schizophrenia } \\
\mathrm{N}=13,697\end{array}$ & $\begin{array}{l}\text { Patients without } \\
\text { schizophrenia } \\
N=13,697\end{array}$ & $\begin{array}{l}\text { Standardized } \\
\text { difference, \% }\end{array}$ \\
\hline \multicolumn{7}{|l|}{ Year of index date, $\mathrm{n}(\%)$} \\
\hline 1998-2002 & $1,417 \quad(7.5)$ & $1,202 \quad(6.4)$ & 4.5 & $1,525(11.1)$ & $951 \quad(6.9)$ & 14.7 \\
\hline $2003-2007$ & $2,918(15.5)$ & 2,319 (12.4) & 9.2 & $2,781(20.3)$ & $1,948(14.2)$ & 16.1 \\
\hline $2008-2012$ & $6,297(33.5)$ & $6,210(33.1)$ & 1.0 & $4,036(29.5)$ & $3,966(29.0)$ & 1.1 \\
\hline $2013-2017$ & $8,139(43.4)$ & $9,040(48.2)$ & 9.6 & 5,355 (39.1) & $6,832(49.9)$ & 21.8 \\
\hline $\begin{array}{l}\text { Quan-CCI, mean } \pm \mathrm{SD} \\
\text { [median; Q1-Q3] }\end{array}$ & $\begin{array}{c}2.4 \pm 2.6 \\
{[2.0 ; 0.0-4.0]}\end{array}$ & $\begin{array}{c}1.7 \pm 2.3 \\
{[1.0 ; 0.0-3.0]}\end{array}$ & 26.9 & $\begin{array}{c}2.2 \pm 2.6 \\
{[1.0 ; 0.0-3.0]}\end{array}$ & $\begin{array}{c}1.5 \pm 2.3 \\
{[1.0 ; 0.0-2.0]}\end{array}$ & 27.2 \\
\hline \multicolumn{7}{|l|}{ Additional comorbidities, n (\%) } \\
\hline Diabetes & $6,220(33.1)$ & $4,322(23.0)$ & 22.6 & 4,015 (29.3) & $2,847(20.8)$ & 19.8 \\
\hline Hypertension & $10,908(58.1)$ & $7,848(41.8)$ & 33.0 & $7,249(52.9)$ & $5,198 \quad(37.9)$ & 30.4 \\
\hline $\begin{array}{l}\text { Unique mental health diagno- } \\
\text { ses, mean } \pm \text { SD [median; Q1-Q3] }\end{array}$ & $\begin{array}{c}8.0 \pm 5.4 \\
{[7.0 ; 4.0-11.0]}\end{array}$ & $\begin{array}{c}1.2 \pm 2.0 \\
{[0.0 ; 0.0-2.0]}\end{array}$ & 168.3 & $\begin{array}{c}8.5 \pm 5.4 \\
{[7.0 ; 5.0-11.0]}\end{array}$ & $\begin{array}{c}1.2 \pm 1.9 \\
{[0.0 ; 0.0-2.0]}\end{array}$ & 179.6 \\
\hline $\begin{array}{l}\text { Pharmacy costs, mean } \pm \text { SD } \\
\text { [median; Q1-Q3], \$US } 2018\end{array}$ & $\begin{array}{l}4,322 \pm 9,344 \\
{[228 ; 0-5,019]}\end{array}$ & $\begin{array}{c}2,082 \pm 8,752 \\
{[29 ; 0-743]}\end{array}$ & 24.7 & $\begin{array}{c}5,450 \pm 9,708 \\
{[2,413 ; 369-7,431]}\end{array}$ & $\begin{array}{c}2,521 \pm 10,282 \\
{[62 ; 0-1,095]}\end{array}$ & 29.3 \\
\hline $\begin{array}{l}\text { Medical costs, mean } \pm \text { SD } \\
\text { [median; Q1-Q3], \$US } 2018\end{array}$ & $\begin{array}{c}37,301 \pm 49,160 \\
{[19,370} \\
5,170-53,873]\end{array}$ & $\begin{array}{c}17,488 \pm 42,245 \\
{[1,865} \\
191-13,348]\end{array}$ & 43.2 & $\begin{array}{c}39,871 \pm 50,434 \\
{[24,097} \\
10,540-50,483]\end{array}$ & $\begin{array}{c}16,742 \pm 42,093 \\
{[1,880 ;} \\
206-11,755]\end{array}$ & 49.8 \\
\hline
\end{tabular}

aPatients with and without schizophrenia in the overall population were matched 1:1 using exact matching factors (ie, age groups) and propensity scores generated based on sex, race, state, insurance eligibility, and year of the index date. No subgroup-specific propensity scores were generated.

$\mathrm{CCl}=$ Charlson comorbidity index; $\mathrm{Q} 1$ = first quartile; $\mathrm{Q} 3=$ third quartile; $\mathrm{SD}=$ standard deviation .

Costs were assessed from the Medicaid payers perspective and were reported in 2018 US dollars after adjusting for inflation using the medical care component of the US Consumer Price Index.

Statistical Analysis. Baseline characteristics were described in matched pairs of patients with and without schizophrenia using means, standard deviations (SDs), medians, and interquartile ranges (IQRs) for continuous variables, and frequencies and proportions for categorical variables. The balance of baseline characteristics between matched pairs was evaluated using standardized differences $(<10 \%$ considered well-balanced). ${ }^{13}$

Outcomes were compared between matched pairs with and without schizophrenia using regression models. Multivariate adjustments for baseline characteristics that became unbalanced after matched pairs were subset from the matched cohorts with and without schizophrenia were included in regression models. To compare HRU, Poisson regression models with generalized estimating equations
(GEEs) to account for matched pairs were used to generate incidence rate ratios (IRRs). Similarly, costs were compared using ordinary least squares regression models with GEEs to generate mean differences. Non-parametric bootstrap procedures were used to generate $95 \%$ confidence intervals (CIs) and P values associated with IRRs and mean cost differences.

\section{Results}

\section{BASELINE CHARACTERISTICS}

Among 158,763 patients with schizophrenia, 18,771(11.8\%) had $\geq 1$ schizophrenia-related inpatient or ED claim in the 60 days prior to or on the index date (recent relapse; Figure 1). The mean age was $50.5(\mathrm{SD}=16.3)$ years, $48.6 \%$ were female, and $51.4 \%$ were male (Table 1). Baseline demographic characteristics were well-balanced between patients with recently relapsed schizophrenia and their controls without schizophrenia. Patients with recently relapsed schizophrenia 


\section{FIGURE 2 All-Cause HRU During the 12-Month Observation Period in Patients with Recently Relapsed} Schizophrenia Relative to Patients Without Schizophrenia a,b

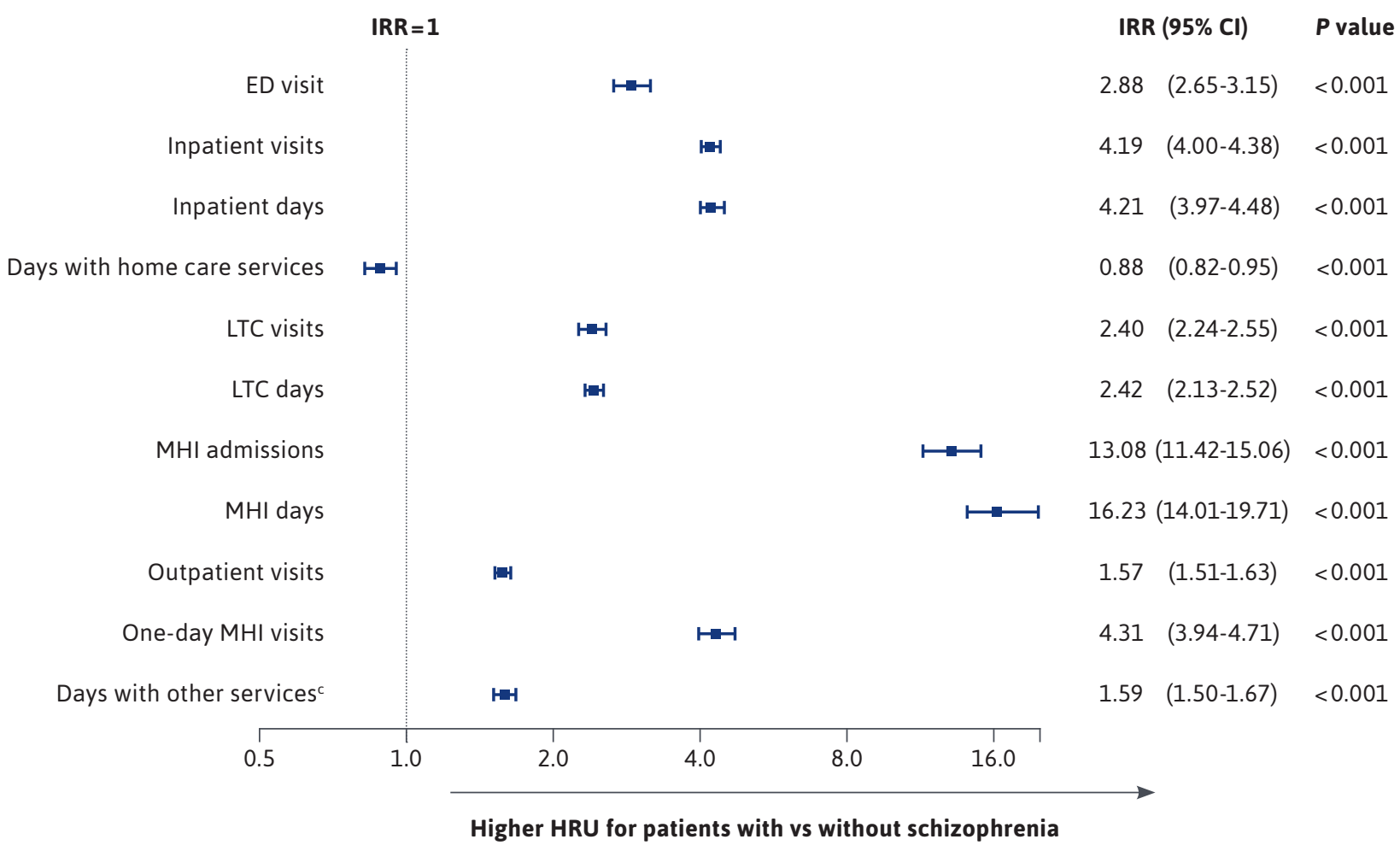

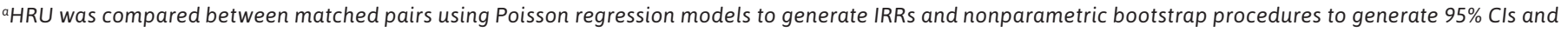
$P$ values.

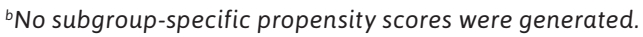

'Other services include but are not limited to independent laboratory services and services received in Federally Qualified Health Centers.

$\mathrm{Cl}=$ confidence interval; $\mathrm{ED}=$ emergency department; $H R U=$ healthcare resource utilization; IRR=incidence rate ratio; $L T C=$ long-term $c a r e ; M H I=$ mental health institute.

had a higher prevalence of diabetes (33.1\% vs 23.0\%) and hypertension (58.1\% vs $41.8 \%)$, as well as a higher number of unique baseline mental health diagnoses ( $8.0 \mathrm{vs} 1.2)$ than their controls. Total baseline healthcare costs were higher among patients with recently relapsed schizophrenia than their controls without schizophrenia (\$41,623 vs \$19,570), including higher medical (\$37,301 vs $\$ 17,488)$ and pharmacy costs $(\$ 4,322$ vs $\$ 2,082)$.

A cohort of 13,697 (8.6\%) patients had uncontrolled symptoms of schizophrenia and were not adherent to APs during the baseline period (Figure 1). The mean age among these patients was 47.1 years $(\mathrm{SD}=15.4), 48.0 \%$ were female, and $52.0 \%$ were male (Table 1). Patients with uncontrolled symptoms of schizophrenia also had a higher prevalence of diabetes (29.3\% vs $20.8 \%$ ) and hypertension $(52.9 \%$ vs $37.9 \%)$, as well as a higher number of unique baseline mental health diagnoses (8.5 vs 1.2$)$ than their controls. Total baseline healthcare costs were higher among non-adherent patients with uncontrolled symptoms of schizophrenia than their controls without schizophrenia (\$45,320 vs \$19,262), including higher medical (\$39,871 vs $\$ 16,742)$ and pharmacy costs $(\$ 5,450$ vs $\$ 2,521)$.

\section{BURDEN OF RECENTLY RELAPSED SCHIZOPHRENIA}

During the 12-month observation period, patients with recently relapsed schizophrenia had higher HRU relative to controls without schizophrenia, including 4.21 times more inpatient days (mean: 25.1 vs 6.0 days, respectively), 2.88 times more ED visits (mean: 3.4 vs 1.2 visits, respectively), 1.57 times more outpatient visits (mean: 13.3 vs 8.5 visits, respectively), 4.31 times more one-day MHI visits (mean: 4.3 vs 1.0 visits, respectively), 2.42 times more LTC days (mean: 78.7 
FIGURE 3 All-Cause Healthcare Costs During the 12-Month Observation Period in Patients with Recently Relapsed Schizophrenia Relative to Patients Without Schizophrenia ${ }^{\mathrm{a}, \mathrm{b}}$ and in Non-Adherent Patients with Uncontrolled Symptoms of Schizophrenia Relative to Patients Without Schizophreniab,c

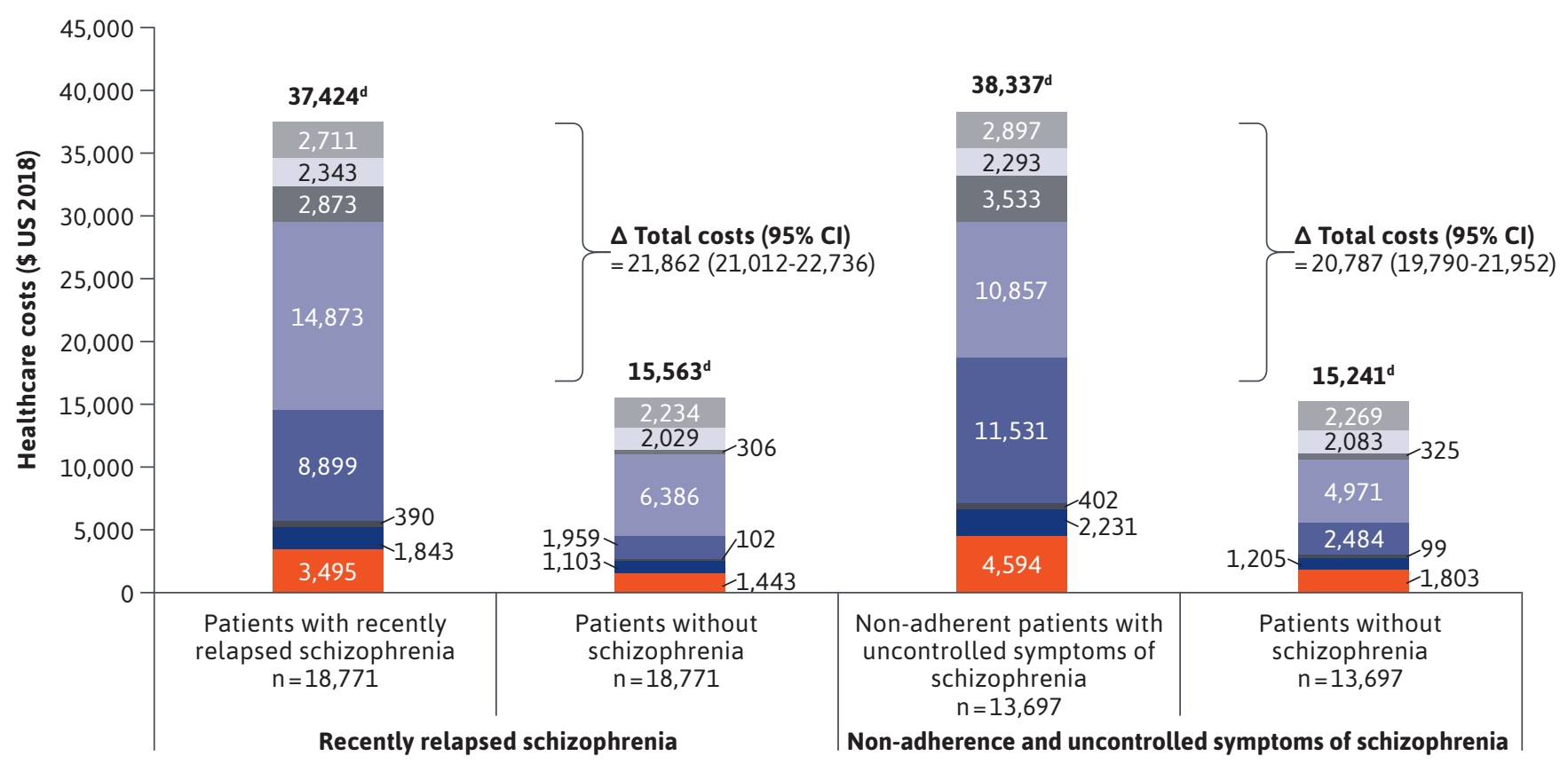

Pharmacy $\square$ Outpatient $\square$ ED Inpatient $\square$ LTC $\square$ MHI (including one-day visits) $\square$ Home care $\square$ Other $^{c}$

${ }^{a}$ Costs were compared between matched pairs using ordinary least squares regression models to generate mean differences and nonparametric bootstrap procedures to generate $95 \% \mathrm{Cl}$ and $\mathrm{P}$ values.

${ }^{b}$ No subgroup-specific propensity scores were generated.

'Costs were compared between matched pairs using ordinary least squares regression models, adjusting for unbalanced matching factors, which included race, state, year of the index date, and insurance eligibility, to generate mean differences and nonparametric bootstrap procedures to generate $95 \% \mathrm{Cls}$ and $\mathrm{P}$ values. Individual cost components do not sum to the total cost because of rounding.

e Other services include but are not limited to independent laboratory services and services received in Federally Qualified Health Centers.

$\mathrm{Cl}=$ confidence interval; $\mathrm{ED}=$ emergency department; $\mathrm{LTC}=$ long-term care; $\mathrm{MHI}=$ mental health institute; US=United States

vs 32.6 days, respectively), and 16.23 times more MHI days (mean: 21.7 vs 1.3 days, respectively; all $\mathrm{P}<0.001$; Figure 2).

The higher all-cause HRU among patients with recently relapsed schizophrenia was predominantly driven by mental health- and schizophrenia-related HRU. In terms of mental health-related HRU, patients with recently relapse schizophrenia had 12.26 times more inpatient days (mean: 22.1 vs 1.8 days, respectively), 12.23 times more ED visits (mean: 1.3 vs 0.1 visits, respectively), 3.02 times more outpatient visits (mean: 5.6 vs 1.8 visits, respectively), 3.66 times more LTC days (mean: 71.8 vs 19.6 days, respectively), and 17.58 times more MHI days compared to matched controls without schizophrenia (mean: 21.0 vs 1.2 days, respectively; all $\mathrm{P}<0.001$;
Supplementary Figure 2, available in online article). In terms of schizophrenia-related HRU, patients with recently relapsed schizophrenia had a mean of 17.5 inpatient days, 2.3 outpatient visits, 2.1 one-day MHI visits, 60.0 LTC days, and 14.7 MHI days during the observation period.

All-cause mean total healthcare costs were $\$ 21,862$ higher among patients with recently relapsed schizophrenia relative to controls without schizophrenia $(\$ 37,424$ vs $\$ 15,563$, respectively; $P<0.001 ;$ Figure 3). This difference was largely due to $\$ 8,486$ higher mean LTC, $\$ 6,940$ higher mean inpatient, \$2,225 higher mean MHI, as well as \$2,052 higher mean pharmacy costs among patients with recently relapsed schizophrenia (all $\mathrm{P}<0.001$ ). 


\section{FIGURE 4 All-Cause HRU During the 12-Month Observation Period in Non-Adherent Patients with Uncontrolled Symptoms of Schizophrenia Relative to Patients Without Schizophrenia ${ }^{a, b}$}

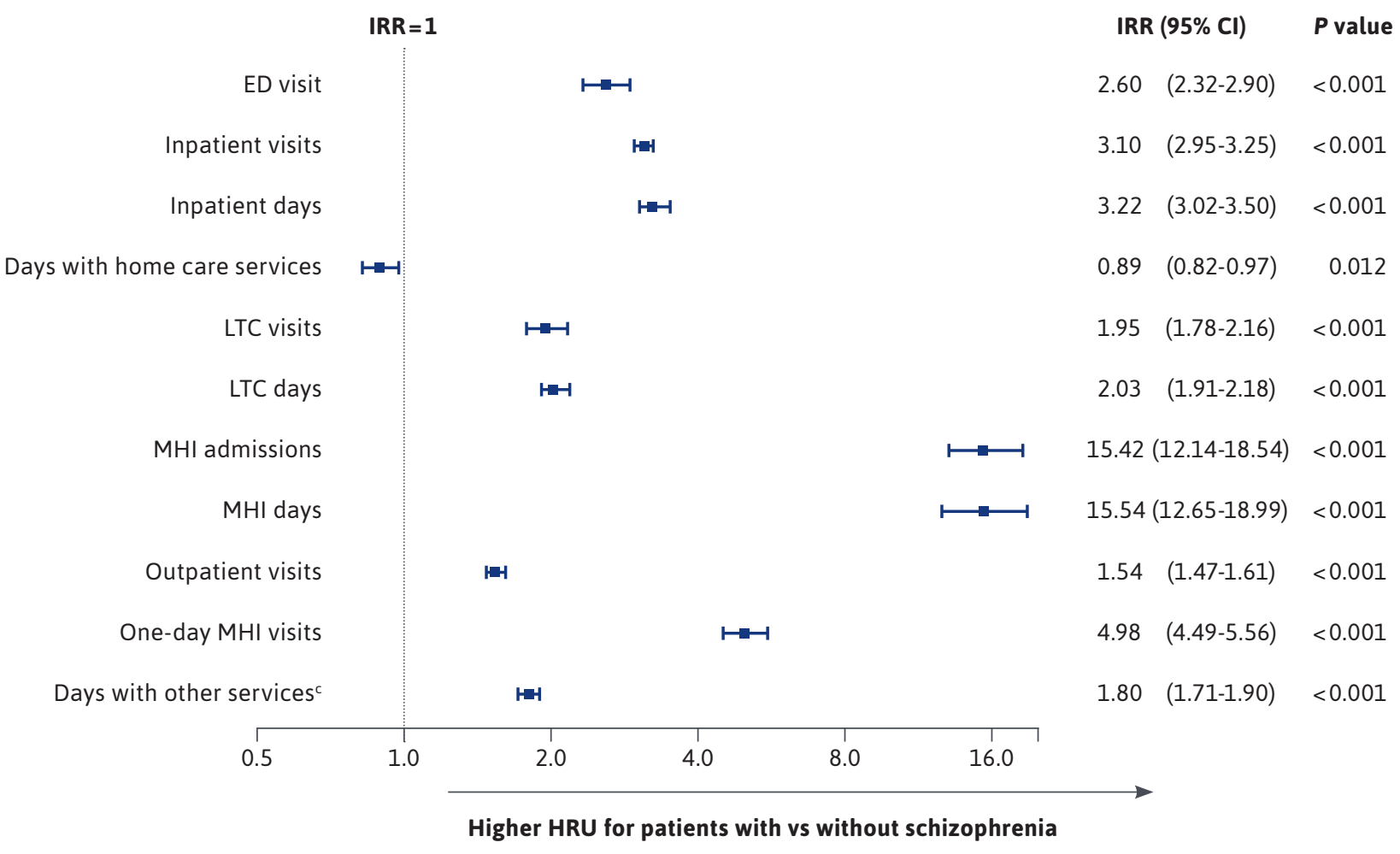

${ }^{a} \mathrm{HRU}$ was compared between matched pairs using Poisson regression models, adjusting for unbalanced matching factors, which included race, state, insurance eligibility, and year of the index date to generate IRRs and nonparametric bootstrap procedures to generate $95 \%$ Cls and $P$ values.

${ }^{b}$ No subgroup-specific propensity scores were generated.

'Other services include but are not limited to independent laboratory services and services received in Federally Qualified Health Centers.

$\mathrm{Cl}=$ confidence interval; $\mathrm{ED}=$ emergency department; $H R U=$ healthcare resource utilization; $I R R=$ incidence rate ratio; $L T C=$ long-term $c$ are; $M H I=$ mental health institute.

Similar to HRU, the higher all-cause mean total healthcare costs among patients with recently relapsed schizophrenia were predominantly driven by mental health-related and schizophrenia-related costs. Mental health-related mean costs were $\$ 16,374$ higher among patients with recently relapsed schizophrenia relative to controls without schizophrenia ( $\$ 21,133$ vs $\$ 4,760$, respectively; $P<0.001$; Supplementary Figure 3, available in online article) and accounted for $74.9 \%$ of the all-cause mean total healthcare cost difference. Schizophrenia-related mean total healthcare costs were $\$ 12,388$ among patients with recently relapsed schizophrenia.

\section{BURDEN OF UNCONTROLLED SYMPTOMS OF SCHIZOPHRENIA WITH NON-ADHERENCE TO AP THERAPY}

During the 12-month observation period, non-adherent patients with uncontrolled symptoms of schizophrenia had higher HRU relative to controls without schizophrenia, including 3.22 times more inpatient days (mean: 23.4 vs 6.5 days, respectively), 2.60 times more ED visits (mean: 3.1 vs 1.1 visits, respectively), 1.54 times more outpatient visits (mean: 13.1 vs, 8.6 visits, respectively), 4.98 times more one-day MHI visits (mean: 5.8 vs 1.2 visits, respectively), 2.03 times more LTC days (mean: 53.7 vs 24.0 days, respectively), and 15.54 times more MHI days (mean: 19.1 vs 1.4 days, respectively; all $\mathrm{P}<0.001$; Figure 4). 
The higher all-cause HRU among non-adherent patients with uncontrolled symptoms of schizophrenia was predominantly driven by mental health- and schizophrenia-related HRU. In terms of mental health-related HRU, non-adherent patients with uncontrolled symptoms of schizophrenia had 9.77 times more inpatient days (mean: 20.2 vs 2.0 days, respectively), 11.01 times more ED visits (mean: 0.8 vs 0.1 visits, respectively), 2.59 times more outpatient visits (mean: 5.9 vs 2.2 visits, respectively), 3.15 times more LTC days (mean: 47.8 vs 14.2 days, respectively), and 17.66 times more MHI days compared to controls without schizophrenia (mean: 18.6 vs 1.2 days, respectively; all $\mathrm{P}<0.001$; Supplementary Figure 4, available in online article). In terms of schizophrenia-related HRU, non-adherent patients with uncontrolled symptoms of schizophrenia had a mean of 15.1 inpatient days, 1.9 outpatient visits, 2.9 one-day MHI visits, 38.4 LTC days, and 12.0 MHI days during the observation period.

The all-cause mean adjusted healthcare cost difference was \$20,787 among non-adherent patients with uncontrolled symptoms of schizophrenia relative to controls without schizophrenia (mean: $\$ 38,337$ vs $\$ 15,241$, respectively; $\mathrm{P}<0.001$; Figure 3). This difference was largely due to \$8,019 higher inpatient, \$4,894 higher LTC, \$2,517 higher MHI, as well as $\$ 2,294$ higher pharmacy costs among non-adherent patients with uncontrolled symptoms of schizophrenia (adjusted mean cost differences; all $\mathrm{P}<0.001$ ).

Similar to HRU, the higher all-cause healthcare costs among non-adherent patients with uncontrolled symptoms of schizophrenia were predominantly driven by mental health-related and schizophrenia-related costs. The mental health-related mean adjusted cost difference among non-adherent patients with uncontrolled symptoms of schizophrenia relative to controls without schizophrenia was $\$ 16,445$ (mean: $\$ 21,731$ vs $\$ 4,677 ; \mathrm{P}<0.001$; Supplementary Figure 3 ) and accounted for $79.1 \%$ of the all-cause mean adjusted healthcare cost difference. Mean schizophreniarelated costs were $\$ 12,411$ among non-adherent patients with uncontrolled symptoms of schizophrenia.

\section{Discussion}

In this six-state Medicaid study, patients with schizophrenia and a recent relapse as well as those not adherent to APs with uncontrolled symptoms of schizophrenia incurred substantial all-cause HRU and costs relative to patients without schizophrenia.

Prior studies have demonstrated that a schizophrenia relapse may increase the cost burden of patients with schizophrenia. ${ }^{11,14}$ Specifically, mean costs of a relapse episode defined using a claims-based algorithm were estimated as $\$ 35,725$ (2011 USD) in a study of Medicaid patients with schizophrenia between 1997 and 2010. ${ }^{11}$ However, to our knowledge, the economic burden of schizophrenia with a recent relapse has not been previously quantified. Indeed, our study showed that patients with recently relapsed schizophrenia incurred additional \$21,862 in mean healthcare costs during the 12-month observation period compared to patients without schizophrenia, which is higher than incremental healthcare costs of all Medicaid patients with schizophrenia, recently reported as a mean of $\$ 14,087$ per patient annually. ${ }^{10}$

This study also evaluated the economic burden of patients who were not adherent to APs and had uncontrolled symptoms of schizophrenia. Non-adherence to AP therapy and uncontrolled symptoms of schizophrenia are highly correlated., ${ }^{45}$ While uncontrolled symptoms along with relapse have been shown to contribute to higher healthcare costs, ${ }^{11,14}$ the link between non-adherence and costs is not as clear. Although an increase in hospitalization costs is typically observed with AP non-adherence, this increase is often offset by decreased pharmacy costs. ${ }^{12}$ The current study found that non-adherent patients with uncontrolled symptoms of schizophrenia incurred $\$ 20,787$ higher mean healthcare costs annually compared to patients without schizophrenia. This cost difference appears to be higher than the cost difference between all patients with and without schizophrenia $(\$ 14,087){ }^{10}$

While relapse is typically associated with high inpatient costs in the literature,,$^{11,16}$ the current study identified LTC as an even more substantial driver of excess mean healthcare costs among patients with recently relapsed schizophrenia (39\% of the total mean cost difference). This finding may be due to the considerable proportion of patients (19\%) being older than 65 years in this subgroup, as older age has been shown to lead to higher expenditures related to LTC services, like nursing homes. ${ }^{17}$ Additionally, mental illness that is difficult to manage, like recently relapsed schizophrenia, may prompt caregivers to rely on nursing home placements for patients with schizophrenia at a younger age because of the lack of long-term alternatives in the US healthcare system. ${ }^{18}$ Indeed, in a nationally representative survey-based study of nursing homes in the United States, the proportion of residents younger than 65 years with a primary diagnosis of mental illness was almost 3 times higher than that of older residents..$^{18,19}$

Inpatient visits were the primary driver of excess mean healthcare costs among non-adherent patients with uncontrolled symptoms of schizophrenia (39\% of the total cost difference) while LTC visits contributed $24 \%$ to the mean cost difference relative to patients without schizophrenia. As mentioned earlier, increased inpatient costs among 
patients with schizophrenia who are not adherent to AP therapy are commonly reported..$^{12,20}$ Of note, patients older than 65 years comprised a lower share $(12 \%)$ of non-adherent patients with uncontrolled symptoms of schizophrenia, which may explain the lower contribution of LTC visits to the mean total healthcare cost difference.

The clinical implications of the present findings are important to consider. While schizophrenia is known to be associated with a large economic burden among Medicaid beneficiaries, ${ }^{6,8,10}$ recent relapse and non-adherence to APs with uncontrolled disease further increase this burden. Disease management options that may improve adherence and reduce the risk of relapse and uncontrolled symptoms could help alleviate high medical costs among patients with schizophrenia. Further research is warranted to confirm these findings and identify options to reduce healthcare costs among patients who have experienced schizophrenia relapse and/or are not adherent to APs and have uncontrolled symptoms.

\section{LIMITATIONS}

This study should be interpreted in light of several limitations. First, the analyses were based on Medicaid data from six states, so the findings may not be generalizable to the overall Medicaid population, patients from states not included in this analysis, or patients without health insurance or with non-Medicaid insurance plans. Furthermore, given heterogeneity between states in terms of income thresholds for Medicaid eligibility, the expansion of Medicaid through the Affordable Care Act, and the extent of services covered by the insurance plan, pooled estimates obtained in this study may be different from estimates for individual states. Given data availability until early 2018, estimates may not reflect the most current treatment landscape for schizophrenia. ${ }^{21}$ Since a high proportion of Medicaid beneficiaries included in this study had dual Medicaid/Medicare coverage, the estimated HRU and cost burden may be conservative. Schizophrenia relapse using a clinical definition could not be ascertained within the claims data; therefore, schizophrenia-related inpatient or ED visits were used as a proxy for relapse, and uncontrolled symptoms of schizophrenia were defined as schizophrenia-related hospitalization. Several clinical studies have defined relapse as increased symptom severity and/or psychiatric hospitalization, ${ }^{22-24}$ and relapse has been associated with higher all-cause and psychiatric or schizophrenia-related hospitalization. ${ }^{25}$ However, these proxies have not been validated clinically with another data source. Schizophreniarelated claims were identified based on a diagnosis of schizophrenia in any position and thus may not always represent a clinical encounter for relapse or uncontrolled symptoms. Patients with recently relapsed schizophrenia and non-adherent patients with uncontrolled symptoms of schizophrenia were not re-matched to schizophrenia-free controls with the use of subgroup-specific propensity scores. Since propensity score matching was based on the probability of belonging to the overall schizophrenia cohort, baseline characteristics may have remained unbalanced between the subgroups and their controls. However, comparisons of HRU and costs were adjusted for such unbalanced baseline characteristics. Despite the use of the matching techniques and adjustment for baseline characteristics in regression models, results may be influenced by residual confounding due to unmeasured confounders (ie, information not available in claims data).

\section{Conclusions}

Medicaid beneficiaries with schizophrenia who experienced a recent relapse and those non-adherent to AP therapy with uncontrolled symptoms of schizophrenia incurred significantly higher HRU and healthcare costs compared to Medicaid beneficiaries without schizophrenia. Additional healthcare costs incurred by these subgroups of patients with schizophrenia appeared to exceed the incremental costs incurred by all patients with schizophrenia. These results emphasize the need to optimize disease management in patients with schizophrenia, with the goal of increasing adherence, preventing relapses, and improving symptom control.

\section{DISCLOSURES}

This study was supported by Janssen Scientific Affairs, LLC. The sponsor was involved in the study design, data collection, data analysis, manuscript preparation, and publication decisions.

Pilon, Lafeuille, Zhdanava, Côté-Sergent, Rossi, and Lefebvre are employees of Analysis Group, Inc., a consulting company that has provided paid consulting services to Janssen Scientific Affairs, LLC, which funded the development and conduct of this study and manuscript. Patel, Joshi, and Lin are employees of Janssen Scientific Affairs, LLC and stockholders of Johnson \& Johnson

Part of the material in this manuscript has been presented at the US Psych Congress, October 3-6, 2019, San Diego, CA, and at the Virtual ISPOR Meeting, May $18-20,2020$

\section{ACKNOWLEDGEMENTS}

Medical writing assistance was provided by Mona Lisa Chanda and Christine Tam, employees of Analysis Group, Inc. 


\section{REFERENCES}

1. Schizophrenia. National Institute of Mental Health. Published May 2018. Accessed April 7, 2020. https://www. nimh.nih.gov/health/statistics/schizophrenia.shtml\#part_154880

2. Wu EQ, Shi L, Birnbaum H, Hudson T, Kessler R. Annual prevalence of diagnosed schizophrenia in the USA: a claims data analysis approach. Psychol Med. 2006;36(11):1535-40.

3. Haddad PM, Brain C, Scott J. Nonadherence with antipsychotic medication in schizophrenia: challenges and management strategies. Patient Relat Outcome Meas. 2014;5:43-62.

4. Emsley R, Chiliza B, Asmal L, Harvey BH. The nature of relapse in schizophrenia. BMC Psychiatry. 2013;13:50.

5. Kane JM. Treatment strategies to prevent relapse and encourage remission. J Clin Psychiatry. 2007;68 Suppl 14:27-30.

6. Cloutier M, Aigbogun MS, Guerin A, et al. The Economic Burden of Schizophrenia in the United States in 2013. J Clin Psychiatry. 2016;77(6):764-71.

7. Desai P, Lawson K, Barner J, Rascati K. Estimating the direct and indirect costs for community-dwelling patients with schizophrenia. Journal of Pharmaceutical Health Services Research. 2013;4:187-94.

8. Wu EQ, Birnbaum HG, Shi L, et al. The economic burden of schizophrenia in the United States in 2002. J Clin Psychiatry. 2005;66(9):1122-29.

9. Masand PS, Roca M, Turner MS, Kane JM. Partial adherence to antipsychotic medication impacts the course of illness in patients with schizophrenia: a review. Prim Care Companion J Clin Psychiatry. 2009;11(4):147-54.
10. Lafeuille $\mathrm{MH}$, Patel C, Pilon D, et al. Prevalence, Incidence, and Economic Burden of Schizophrenia among Medicaid Beneficiaries. Paper presented at: Psych Congress 2019; San Diego, CA.

11. Lafeuille MH, Gravel J, Lefebvre P, et al. Patterns of relapse and associated cost burden in schizophrenia patients receiving atypical antipsychotics. J Med Econ. 2013;16(11):1290-9.

12. Pennington M, McCrone P. Does NonAdherence Increase Treatment Costs in Schizophrenia? Pharmacoeconomics. 2018;36(8):941-55.

13. Austin PC. Using the standardized difference to compare the prevalence of a binary variable between two groups in observational research. Communications in Statistics-Simulation and Computation. 2009;38(6):1228-34.

14. Pennington $\mathrm{M}, \mathrm{McCrone} \mathrm{P}$. The Cost of Relapse in Schizophrenia. Pharmacoeconomics. 2017;35(9):921-36.

15. Robinson D, Woerner MG, Alvir JM, et al. Predictors of relapse following response from a first episode of schizophrenia or schizoaffective disorder. Arch Gen Psychiatry. 1999;56(3):241-47.

16. Ascher-Svanum H, Zhu B, Faries DE, et al. The cost of relapse and the predictors of relapse in the treatment of schizophrenia. BMC Psychiatry. 2010;10:2.

17. Bartels SJ, Clark RE, Peacock WJ, Dums AR, Pratt SI. Medicare and medicaid costs for schizophrenia patients by age cohort compared with costs for depression, dementia, and medically ill patients. Am J Geriatr Psychiatry. 2003;11(6):648-57.

18. Donovan K, Regehr C, George M. Nursing Home Care for Adults With Chronic Schizophrenia. Social Work in Mental Health. 2013;11:167-83.
19. Bagchi AD, Verdier JM, Simon SE. How many nursing home residents live with a mental illness? Psychiatr Serv. 2009;60(7):958-64.

20. Hansen RA, Maciejewski M, Yu-Isenberg K, Farley JF. Adherence to antipsychotics and cardiometabolic medication: association with health care utilization and costs. Psychiatr Serv. 2012;63(9):920-28.

21. Correll CU. Current treatment options and emerging agents for schizophrenia. J Clin Psychiatry. 2020;81(3).

22. Chen EY, Hui CL, Lam MM, et al. Maintenance treatment with quetiapine versus discontinuation after one year of treatment in patients with remitted first episode psychosis: randomised controlled trial. BMJ. 2010;341:c4024.

23. Durgam S, Earley W, Li R, et al. Long-term cariprazine treatment for the prevention of relapse in patients with schizophrenia: A randomized, doubleblind, placebo-controlled trial. Schizophr Res. 2016;176(2-3):264-71.

24. Kishi T, Ikuta T, Matsui Y, et al. Effect of discontinuation $v$. maintenance of antipsychotic medication on relapse rates in patients with remitted/stable first-episode psychosis: a meta-analysis. Psychol Med. 2019;49(5):772-9.

25. Addington DE, Patten SB, McKenzie E, Addington J. Relationship between relapse and hospitalization in first-episode psychosis. Psychiatr Serv. 2013;64(8):796-99. 